\title{
Phase coexistence of neutral polymer gels under mechanical constraint
}

\author{
A. Suzuki ${ }^{\mathrm{a})}$ and T. Ishii \\ Department of Materials Science, Yokohama National University, 79-5 Tokiwadai, Hodogaya-ku, \\ Yokohama, 240-8501 Japan
}

(Received 1 July 1998; accepted 29 September 1998)

\begin{abstract}
We have measured the equilibrium diameter of cylindrical poly ( $N$-isopropylacrylamide) (NIPA) gels with submillimeter diameters under a mechanical constraint along the uniaxial direction. The linear swelling curve, diameter vs temperature, was obtained in the vicinity of the volume phase transition temperature under isometric constraint: both ends of the gel were fixed at rest (stress was zero) in a swollen state at $30^{\circ} \mathrm{C}$ (a few degrees below the transition temperature). Thereafter, the temperature was gradually changed by keeping the uniaxial length constant (to the fixed length). It was demonstrated that the gel could take a coexistent state at the transition point and remain stable for several days. This time was much longer than the characteristic relaxation time of the phase transition in this tiny gel. The phase coexistence observed here was caused by stress inhomogeneity along the uniaxial direction due to the mechanical constraint. A collapsed to swollen phase transition induced by uniaxial stress at a fixed temperature was also presented. It was shown that the ratio of the swollen portion to the total length could be controlled by the degree of elongation: when the elongation was slightly increased or decreased, the phase boundary between the swollen and collapsed phases was accordingly shifted to increase or decrease the swollen state, respectively. The ratio of the swollen to the collapsed phase in the case of stress-induced coexistence is discussed herein in terms of a phase diagram (diameter vs elongation), and a simple phase selection rule is presented. (C) 1999 American Institute of Physics. [S0021-9606(99)51801-1]
\end{abstract}

\section{INTRODUCTION}

Several critical phenomena have been investigated in a wide variety of systems, fluids and solids. ${ }^{1-3}$ Concerning fluid systems, the equation of states can be expressed by the variables, $P$ (pressure), $T$ (temperature), and $\rho$ (density). If the environment conditions of the system are properly selected, a discontinuous phase transition can be observed not only in $P-T$ projection but also $T-\rho$ or $\rho-P$ plane. At the transition point, a coexistent state is expected, and a freeenergy profile can determine the phase behavior. For polymer gels, on the other hand, the phase can be described by the swelling curve (corresponding to $T$ - $\rho$ of a fluid system): this is the change in volume, $V$ as a function of an environment parameter which determines polymer-solvent interaction. ${ }^{4,5}$ Polymer gels are made of cross-linked networks of polymers and liquids (dilute solids with a complex structure). They present solidlike and liquidlike behavior due to their elastic and osmotic nature, respectively. It is well known that such simple-composition gels can exist in two distinct phases, swollen or collapsed, which is characterized by the network density, $\phi$ (corresponding to $\rho$ or $V$ ). Several studies on slightly cross-linked polymer gels have shown a very sharp volume change, which has been considered to be a discontinuous volume phase transition. ${ }^{6,7}$ If the environment condition is properly selected both in $T-\rho$ and $T-V$ or $V-P$ planes, a coexistent state could be expected. In this paper, we discuss the phase coexistence of polymer gels in the $T-V$ or $V-P$ plane.

\footnotetext{
a) Author to whom correspondence should be addressed.
}

Hirokawa and $\mathrm{Tanaka}^{8}$ were the first to report that among phase transition gels, neutral poly( $N$-isopropylacrylamide) (NIPA) gel, which is slightly cross-linked by $N, N^{\prime}$-methylenebisacrylamide (BIS), can exhibit a volume phase transition in water only by changing the temperature. Its mechanism has been attributed to a change in the balance of hydrophilic and hydrophobic interaction: NIPA polymers have well-known hydrophobic properties, and volume phase transition properties are primarily due to hydrophobic effects. The swelling curve is highly reproducible. For example, in our experiments on NIPA gels with submillimeter diameters, ${ }^{9,10}$ the phase transition of this normal gel without constraints could be induced by simply increasing or decreasing the temperature by $0.1{ }^{\circ} \mathrm{C}$ in the vicinity of the transition temperature. This was achieved as long as we could regulate the water temperature with an accuracy of $\pm 0.05^{\circ} \mathrm{C}$. Most experimental evidence on a specific gel is limited within the relation between the environment condition and $\phi$ (or $V$ ) [in $T$ - $\phi$ (or $V$ ) projection at a constant $P$ ]. On the other hand, discontinuous volume change is believed to be a universal phenomenon for any kind of gels, if variables $P, T$, and $\phi$ (or $V$ ) can be properly selected. ${ }^{11,12}$ Therefore, in order to show this universality experimentally, it is important to take into consideration the parameter $P$. So far, two different methods have been reported to change the environment condition, these methods having a similar role changing $P$ in the gel system: one is hydrostatic pressure on the liquid, ${ }^{13,14}$ and the other is elongation or compression of solid polymers. ${ }^{15,16}$ Recently, we carried out experimental research on the static properties of mechanically constrained NIPA gels through phase transition behavior. ${ }^{17,18}$ We mea- 
sured the diameter and force of cylindrical NIPA gels with submillimeter diameters under mechanical distortion along the uniaxial direction in small ${ }^{17}$ and large ${ }^{18}$ elongation ratios. We observed that both the transition temperature and force increased when the fixed length in the small region along the uniaxial direction increased. The elastic response, especially in the case of a smaller deformation, was quite successfully described by the phenomenological model on the basis of extended Flory-type free energy. ${ }^{4,15-18}$ It could be said that the consistency of experimental observation with the meanfield theory resulted from the fact that the deformation was small enough not to disturb the assumption of Gaussian behavior of polymer networks too obviously.

To our knowledge, phase coexistence has been experimentally studied on ionized polymer gels but not on neutral ones. Tanaka et al. ${ }^{19}$ reported the coexistence of swollen and collapsed phases in ionized acrylamide gels. This coexistence can be controlled by a change in electric field. Hirotsu $^{16,20,21}$ observed phase coexistence in ionized NIPA gels at the transition point, determining that this coexistence can be controlled by a temperature change. In the latter case, increasing the ionization enhanced the duration of the temperature range of phase coexistence. These two examples are based on the volume change in $P-T-\phi$ (or $V$ ) space by external fields through microscopic compression or stretching of ionized network chains. If the gels were neutral, the effects of the external fields would disappear (the parameter in the $P$ axis becomes zero).

In this paper, we studied the phase transition behavior of neutral NIPA gels of cylindrical shape with submillimeter diameters under a macroscopic mechanical constraint. It was shown that a mechanical constraint can induce a coexistent state at the transition point, and that the ratio of the swollen to the collapsed phase can be controlled by uniaxial stress. The present investigation is focused on the transition behavior of neutral NIPA gels in the vicinity of the transition temperature in $P-T-\rho$ space.

\section{EXPERIMENTAL METHODS}

Gels were synthesized by a free radical copolymerization reaction in glass microcapillaries with an inner diameter of $141.5 \mu \mathrm{m}$. In this synthesis, the base solution was the standard mixture ${ }^{8,17,18}$ for NIPA gel: $7.8 \mathrm{~g}$ of purified NIPA (main constituent, Kohjin), $133 \mathrm{mg}$ of $N, N^{\prime}$ methylenebisacrylamide (BIS, cross-linker, Wako), and 240 $\mu \mathrm{l}$ of $N, N, N^{\prime}, N^{\prime}$-tetramethylethylenediamine (TEMED, accelerator, Wako) were dissolved in $100 \mathrm{~g}$ water; thereafter the solution was stored at $0^{\circ} \mathrm{C}$. After the solution was fully saturated with nitrogen by bubbling, $40 \mathrm{mg}$ of ammonium persulfate (APS, initiator, Wako) was added to this mixture to initiate the reaction. Immediately after that, the microcapillaries were inserted into the pregel solution. Gelation was carried out at $0{ }^{\circ} \mathrm{C}$ overnight for the complete reaction process. After gelation, cylindrical gels were removed from microcapillaries and subsequently washed in distilled deionized pure water for several days to wash away residual chemicals and unreacted monomers from polymer networks.

The experimental setup was as described in Ref. 18: one end of the dried gel was fixed to the capillary, while a small

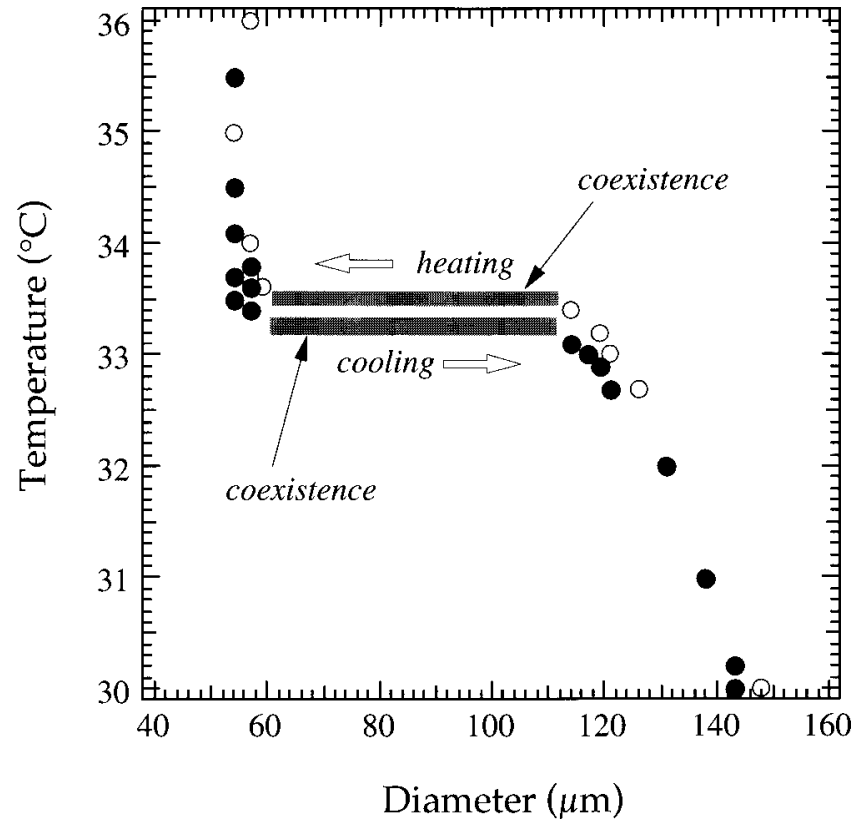

FIG. 1. The equilibrium diameter of the neutral NIPA gels under mechanical constraint as a function of temperature. The gel was isometrically constrained to the equilibrium length at $30^{\circ} \mathrm{C}$; hereafter the temperature was slightly changed. The shaded range on heating or cooling denotes the temperature range of the phase coexistence.

iron tip was attached on the other end by using silicone glues in both cases. A gel has a uniform cylindrical shape; its effective length, which is not affected by the glue, was determined by excluding the attachment parts. Note that the effective length of the gels, which is much larger than the diameter, is over one millimeter. The gel was inserted into a rectangular glass tube with an inner size of $5 \times 5 \mathrm{~mm}^{2}$ filled with pure water so that the iron tip could be fixed to the magnet. The rectangular glass tube was encapsulated in a larger transparent cell. Temperature-controlled water with an accuracy of $\pm 0.05^{\circ} \mathrm{C}$ was circulated in the cell. The temperature was gradually increased, decreased, or fixed in the vicinity of the volume phase transition temperature. After the gel reached equilibrium at each temperature, the diameter of the gels and the effective length of the swollen portion in the coexistence state were then measured by a calibrated CCD microscope apparatus. A load cell was used to determine the length of the gel which was at rest with zero stress. A 20-min interval was enough for the this tiny gel to reach thermallyswelling equilibrium except near the transition point. ${ }^{9,10,17,18}$

\section{RESULTS AND DISCUSSION}

\section{A. Swelling curve}

First of all, the swelling curve of the gels was determined; both ends of the cylindrical gel were fixed at rest in a swollen state at $30^{\circ} \mathrm{C}$ (a few degrees below the transition temperature) and the uniaxial length was restricted to the fixed length while varying the temperature. By measuring the diameter as a function of temperature, we could estimate the properties of the swelling behavior under a mechanical constraint (isometric). Figure 1 shows the equilibrium diameter 


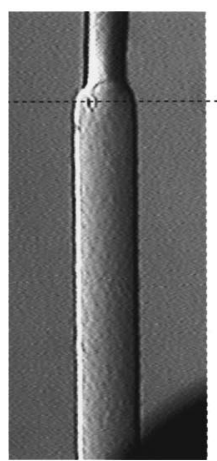

5 hours

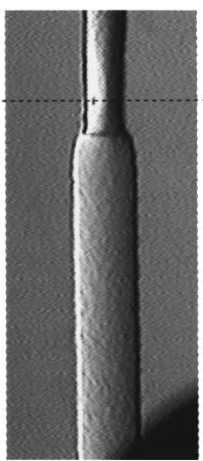

2,3 hours

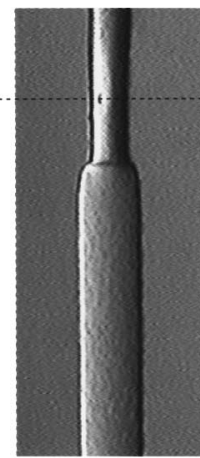

71 hours

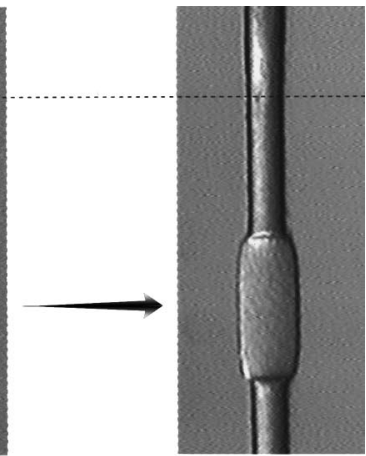

230 hours (a)

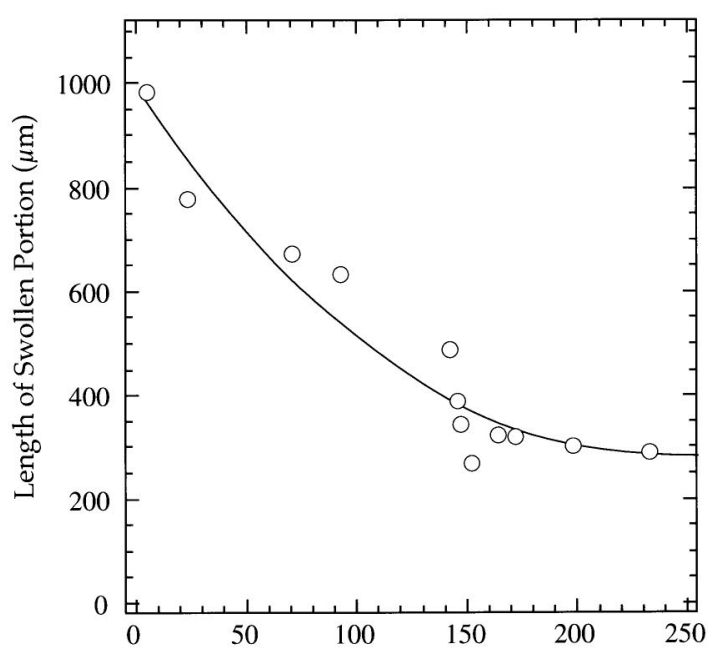

(b)

Time (hr)
FIG. 2. (a) Photographic sequences of the neutral NIPA gel at the transition temperature under mechanical constraint, where the gel is approaching the equilibrium coexistent state with time at $33.5^{\circ} \mathrm{C}$. The gel was isometrically constrained to the equilibrium length at $30^{\circ} \mathrm{C}$, and was in the swollen state at $33.4^{\circ} \mathrm{C}$ on the heating process. The temperature was raised to $33.5^{\circ} \mathrm{C}$ at time zero. The scratch on the surface is connected by the broken line. (b) Time course of the length of the swollen portion at $33.5^{\circ} \mathrm{C}$. Line added is to guide the eye. The experimental error bars are smaller than the symbols. The scattering of data could be attributed to the slight change in the elongation for a long time experiment due to the external factors, such as thermal stability of the sample holder. vs temperature of the neutral NIPA gels under the constraint of the uniaxial length on heating and cooling processes. The temperature was gradually changed in the vicinity of the transition point by $0.1^{\circ} \mathrm{C}$ (minimum step). As mentioned above, the free gel without mechanical constraint could go from the swollen to the collapsed phase only when the temperature was raised by this minimum step, and vice versa in the cooling process. On the other hand, this slightly constrained gel did not finish its transition within this minimum step. In the heating process, the gel that stayed completely in the swollen state at $33.4^{\circ} \mathrm{C}$ did not completely collapse at $33.5^{\circ} \mathrm{C}$ but moved on the phase coexistence, collapsing later at $33.6{ }^{\circ} \mathrm{C}$. In the cooling process, the gel stayed in the collapsed state at $33.3^{\circ} \mathrm{C}$, and then moved on to the phase coexistence, finally swelling at $33.0^{\circ} \mathrm{C}$.

It should be noted that these absolute values for transition temperature and swelling ratios are slightly different between gels prepared from different pregel solutions, but are easily reproducible and exactly the same in the same gel during the repeated temperature cycles. The respective value is the same within the present experimental accuracy when the temperature drift rate or the elongation speed is slow enough not to induce the phase separation. ${ }^{16,22,23}$ The swelling behavior is completely reversible for the same gel.

\section{B. Temperature change}

The stability of the phase coexistence can be shown by slightly increasing the temperature just above the transition point. In the case of the gel shown in Fig. 1 (isometrically constrained to equilibrium length at $30^{\circ} \mathrm{C}$ ), the swollen state starts to collapse by raising the temperature to $33.5^{\circ} \mathrm{C}$. At this temperature, the collapsed portion can emerge at both ends and develop, the gel finally achieving the equilibrium coexistent state. Figure 2(a) shows the evolution of the phase coexistence at $33.5^{\circ} \mathrm{C}$ of the same NIPA gel under an optical microscope. The final state, which is not a transient but a stable or a quasistable equilibrium phenomenon, ${ }^{24}$ consists of the collapsed and swollen states. The phase coexistence disappeared when the temperature was raised further by $0.1^{\circ} \mathrm{C}$ to $33.6{ }^{\circ} \mathrm{C}$. The change in the length of the swollen state is plotted as a function of time in Fig. 2(b). As is shown in this figure, the length of the swollen portion decreased and saturated after around $200 \mathrm{~h}$. It was demonstrated that the gel could remain in a coexistent state at the transition point and be stable for several days. That is much longer than the characteristic relaxation time, ${ }^{9,10,17,18}$ in spite of the critical slowing down at the transition temperature. ${ }^{24}$

Next, the expected contributions of the mechanical constraint on the transition behavior should be considered. In our previous work, ${ }^{17,18}$ it was demonstrated that the uniaxial elongation could increase the transition temperature, which suggests that the macroscopic network elongation can uniquely determine the transition temperature. Therefore, if the stress of the swollen phase can be kept constant, the phase transition can be observed by an infinitesimal temperature increase at the transition point. However, in the present 
experiment, we kept only the total gel length (elongation) constant, and not the strain. Then, if the temperature was increased by a finite degree, the swollen portion locally started to collapse, which induced the stretch of the remaining swollen portion along the uniaxial direction, resulting in an increment of the transition temperature of the remaining portion. This is also related with the fact that the Young modulus of the collapsed state, $E_{C}$, was larger than that of the swollen state, $E_{S}$. The phase coexistence was observed only when heating at $33.5^{\circ} \mathrm{C}$ in the present experiment. This may well be due to the fact that the transition temperature range was too narrow in the $T$-elongation plane to observe the phase coexistence at different temperatures. If we could control the temperature much more precisely, the swollen portion at equilibrium in the phase coexistence would gradually decrease as the temperature increased.

\section{Elongation change}

When the gel in the collapsed state was gradually stretched along the uniaxial direction, a stress-induced swelling phase transition was expected, since the uniaxial stretch could increase the transition temperature. ${ }^{17,18}$ The same NIPA gel used in the above experiment was in the complete collapsed state at $33.5^{\circ} \mathrm{C}$ in the cooling process. By stretching the gel at $33.5^{\circ} \mathrm{C}$, the swollen portion emerged at a threshold elongation which then developed, the gel finally becoming totally swollen at a larger but finite elongation, as shown in Fig. 3(a). It was found that the phase coexistence could be stable if the elongation was stopped along the way. The change in the ratio of the swollen portion to the total length was plotted as a function of the total length, $L$ in Fig. 3 (b). The gel could stay in the phase coexistence, whose mechanism is basically the same as that described in Sec. III B. While stretching along the uniaxial direction, a local portion of the collapsed state might swell discontinuously, inducing the relaxation of the remaining collapsed portion and resulting in a decrement of the transition temperature of the remaining portion. In this case, the difference between the characteristic elongation at the onset and end of the coexistence both in stretching and releasing was large enough to allow us to observe the phase coexistence for many different elongations. If the stretch was drastically increased or decreased, then the gel did not form the phase coexistence, and all stress effects were negated. It should be noted that there was a hysteresis between stretching and releasing, and the length of the first emerged swollen portion had finite length, roughly $100 \mu \mathrm{m}$ (comparable to the diameter). This is related to the fact that the network is continuously connected to form three-dimensional structures. In such systems, even if the embryos or nuclei emerged, they could not stay in the local potential minimum, which is too small to fall into the swollen state by reason of external stress. This is because the energy gain from the network volume change would not exceed the loss of the elastic energy from the boundary strain. Therefore, the swollen portion could emerge only when the swollen portion in the bulk networks developed collectively to have a large enough size in the cross-section of gels. This situation reminds us of the traditional nucleation phenomena $^{25,26}$ in solidification (such as super-cooling),
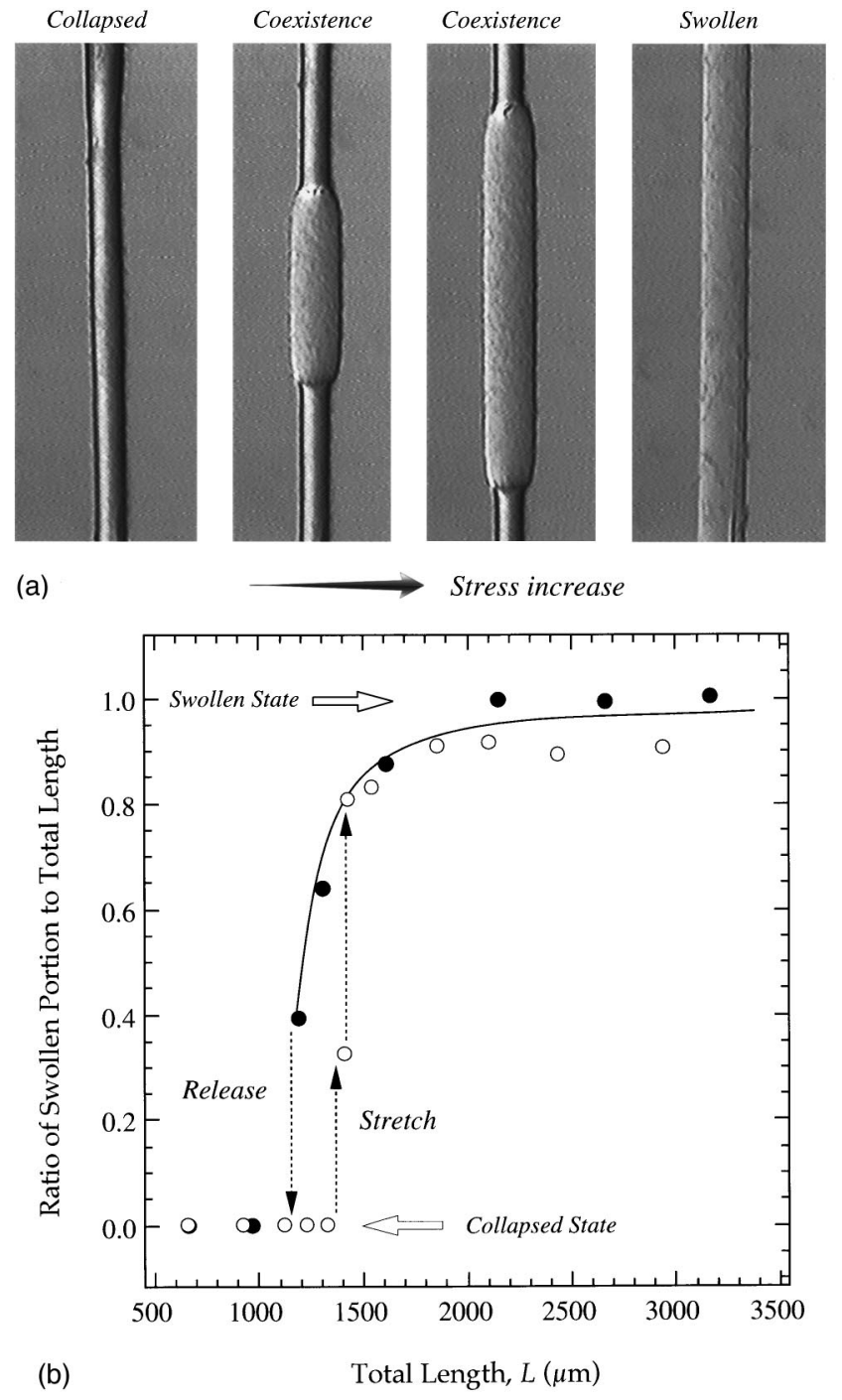

FIG. 3. (a) Photographs of the stress induced phase transition of the NIPA gel, when the gel was gradually stretched along the uniaxial direction. The gel was isometrically constrained to the equilibrium length at $30^{\circ} \mathrm{C}$, and was in the collapsed state at $33.5^{\circ} \mathrm{C}$ on the cooling process. The swollen portion can emerge at a threshold elongation, which develops, and finally the gel takes the swollen state. It should be noted that the phase coexistence is stable, if the elongation was stopped. (b) Change in the ratio of the swollen portion to the total length is plotted as a function of the total length. Line added is to guide the eye. The experimental error bars are smaller than the symbols.

which will be discussed later. From these considerations, the gel, whose uniaxial length is short compared to the diameter, would not exhibit the phase coexistence at the transition point, and the phase transition would finish by the infinitesimal change in the external condition.

\section{Phase diagram of gels under mechanical constraint}

The experimental results can be briefly summarized as follows: during the discontinuous volume phase transition of the neutral polymer gel, if the total length is fixed, the local phase transition can induce the change in the uniaxial strain of the remaining part of the gel (unchanged portion), which stabilize the gel phase as a coexistence. We demonstrate a 

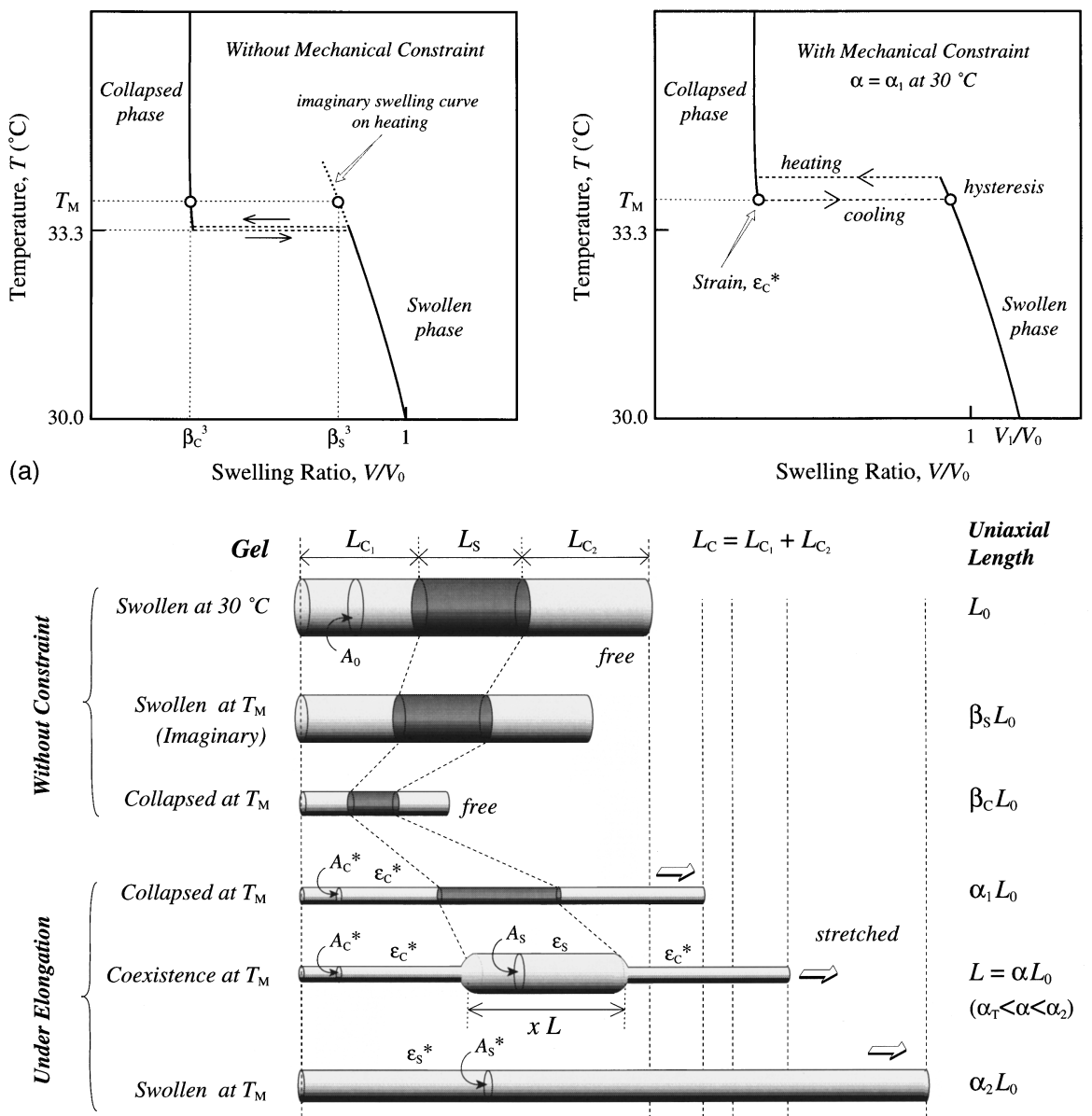

(b)

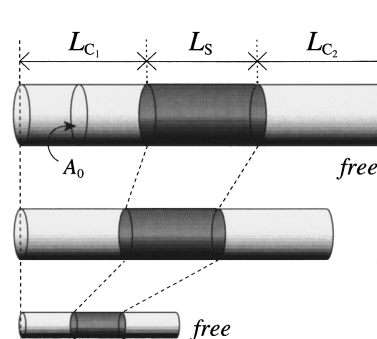

$L_{\mathrm{C}}=L_{\mathrm{C}_{1}}+L_{\mathrm{C}_{2}}$

Uniaxial Length

$L_{0}$

$\beta_{\mathrm{S}} L_{0}$

$\beta_{\mathrm{c}} L_{0}$

$\alpha_{1} L_{0}$

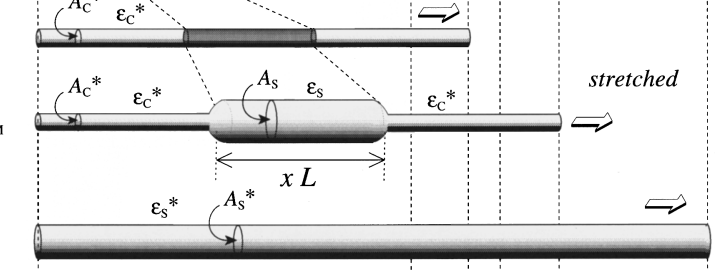

$L=\alpha L_{0}$ $\left(\alpha_{\mathrm{T}}<\alpha<\alpha_{2}\right)$

$\alpha_{2} L_{0}$
FIG. 4. (a) Schematical swelling curves without and with mechanical constraint. The reference states of the free gel without constraint, which is defined to determine the strains of the swollen and the collapsed states in a coexistence, are supposed to have the respective linear swelling ratios, $\beta_{S}$ and $\beta_{C}$. In the latter swelling curve, the gel was elongated by $\alpha_{1}$ at $30^{\circ} \mathrm{C}$ $\left(V_{1}\right.$ denotes the corresponding volume), and was isometrically constrained to the elongated length while changing the temperature. The collapsed to swollen phase transition can occur at $T_{M}$, and the phase coexistence does not occur (see text). (b) Schematic shapes of gel without and with mechanical constraint. A phase coexistence can be realized under an elongation $\alpha\left(\alpha_{T}<\alpha<\alpha_{2}\right)$. The darker areas in the swollen [at $30^{\circ} \mathrm{C}$ and at $T_{M}$ (imaginary)] and the collapsed (at $T_{M}$ ) phases indicate the corresponding swollen portion in the coexistent state. general scheme for controlling the phase coexistence by the uniaxial elongation, where the equilibrium ratio of the swollen portion to the total length, $x$ can be theoretically determined in terms of a simple mechanics as a function of $T$ or $L$. The purpose of this analysis is to find the relation between $x$ and $T$, or $x$ and $L$. The mechanism of the emergence of the phase coexistence is basically the same in each case. In this section, we will describe the latter case, where the gel is gradually stretched along the uniaxial direction at a constant temperature, $T_{M}$ just above the transition point. In this case, we can observe the phase coexistence in a wide range of the variable, $L$.

The longitudinal elongation, $\alpha$ is defined as $L / L_{0}$, that is, the ratio of $L$ to the equilibrium length at rest at $30^{\circ} \mathrm{C}, L_{0}$ (swollen state). It should be noted that the diameter, $d$ of the neutral NIPA gels at $30^{\circ} \mathrm{C}$ without constraint is the same as the inner diameter of the capillary, $d_{0}$ used in the gel synthesis $\left(d / d_{0}=1\right)$, therefore $\alpha=1\left(L=L_{0}\right)$ at $30^{\circ} \mathrm{C} .{ }^{10}$ The swelling ratio, defined as $V / V_{0}$, can be calculated as $\alpha\left(d / d_{0}\right)^{2}$ where $V$ and $V_{0}$ are the volume and the initial volume as prepared, respectively. Figure 4(a) shows schematically the swelling curve ( $T$ vs $V / V_{0}$ ) of the neutral NIPA gel without and with mechanical constraint. The free gel without mechanical constraint could exhibit the phase transition at around $33.4{ }^{\circ} \mathrm{C}$ with a small hysteresis, and take a collapsed state at a target temperature, $T_{M}$. When the gel is isometrically constrained, the phase transition takes place at higher temperatures with a larger hysteresis. We suppose that the collapsed to swollen phase transition could occur at $T_{M}$, when the gel is elongated by $\alpha=\alpha_{1}(>1)$ at $30^{\circ} \mathrm{C}$, and that the gel exhibits the phase transition by a minimum temperature step (without exhibiting the phase coexistence). In the case of this "ideal" gel, the collapsed to swollen phase transition could take place at the critical strain of the collapsed phase, $\epsilon_{C}=\epsilon_{C}^{*}$ [the reference state (the initial state before elongation) is the collapsed state at $T_{M}$ without constraint], and the corresponding cross-sectional area is $A_{C}^{*}$. This ideal one-step phase transition could be realized if the following two conditions were satisfied: one is that the uniaxial length is small enough compared to the diameter not to stabilize the gel phase as a coexistence, and the other is that the nucleation of the new phase can develop in the cross-section under a critical elongation, $\alpha_{1}$ with the strain $\epsilon_{C}^{*}$. In the "actual" case of the present experiment, however, the uniaxial length is much larger than the diameter, and the swollen portion can emerge at a larger elongation $\alpha_{T}\left(>\alpha_{1}\right)$ by increasing $\alpha$ at $T_{M}$. The difference between $\alpha_{T}$ and $\alpha_{1}$ could be attributed to the excess free energy for the nuclei to develop and finally to form the new phase, since the gel has a finite diameter much larger than the size of nuclei (see Sec. IIIE). The swollen portion extends the whole gel if the elongation is further increased. Finally, the gel stays in the single swollen state at the characteristic elongation, $\alpha_{2}$ [see Fig. 4(b)]. At an equi- 
librium phase coexistence at $T_{M}$ under an elongation $\alpha\left(\alpha_{T}\right.$ $<\alpha<\alpha_{2}$ ), the force along the uniaxial direction could be balanced as follows

$$
E_{S} \epsilon_{S} A_{S}=E_{C} \epsilon_{C}^{*} A_{C}^{*},
$$

where $\epsilon_{S}$ denotes the strain of the swollen state in the coexistence (the reference state is the imaginary swollen state at $T_{M}$ without constraint), and the corresponding crosssectional area is $A_{S}$. Note that after an elongation $\alpha\left(\alpha_{T}\right.$ $\left.<\alpha<\alpha_{2}\right)$ is applied within a finite time, $\epsilon_{C}$ decreases to become the critical strain, $\epsilon_{C}^{*}$ with time, and the crosssectional area increases to become the critical value, $A_{C}^{*}$. On the other hand, $\epsilon_{S}$ decreases to approach a characteristic strain $\epsilon_{S}^{*}$ with $\alpha$, finally $\epsilon_{S}=\epsilon_{S}^{*}$ at $\alpha=\alpha_{2}$, while $A_{S}$ increases to become $A_{S}^{*}$ at $\alpha=\alpha_{2}$. The swelling condition could be expressed as

$$
L_{0}=L_{S}+L_{C}=\frac{x L}{\beta_{S}\left(\epsilon_{S}+1\right)}+\frac{(1-x) L}{\beta_{C}\left(\epsilon_{C}^{*}+1\right)},
$$

where $L_{S}$ and $L_{C}$ represent the corresponding equilibrium lengths at rest at $30^{\circ} \mathrm{C}$ (swollen state) of the swollen and collapsed portion in the coexistence at $T_{M}$, respectively, and $\beta_{S}$ and $\beta_{C}$ are the linear swelling ratios of the reference states for calculating the respective strain at $T_{M}$. For the swollen portion, the reference state for elongation is a swollen phase at $T_{M}$ without elongation of the ideal gel (imaginary), while for the collapsed portion it is a collapsed phase at $T_{M}$ without elongation.

In order to obtain the relation between $x$ and $L$, it is necessary to determine the elongation dependence of $A_{S}$. In fact, our recent studies ${ }^{17,18}$ indicated that the uniaxial elongation would increase the swelling ratio of the swollen states, which has not been completely understood at present time (see Sec. IIIE). In the present model, we suppose that the total volume of the swollen state is constant with respect to the uniaxial elongation, $\alpha$,

$$
A_{S} x L=A_{0} L_{S},
$$

where $A_{0}$ is the equilibrium cross-sectional area at rest at $30^{\circ} \mathrm{C}$. From Eqs. (1-3), $\epsilon_{S}$ can be uniquely determined by using the materials constants: $\epsilon_{S}=\left\{\left(E_{S} A_{0}\right) /\left(E_{C} \beta_{S} \epsilon_{C}^{*} A_{C}^{*}\right)\right.$ $-1\}^{-1}$. Therefore, we have the relation between $x$ and $L$ as follows

$$
\begin{aligned}
x & =\left\{1-\frac{\beta_{C}\left(\epsilon_{C}^{*}+1\right)}{\beta_{S}\left(\epsilon_{S}+1\right)}\right\}^{-1}\left\{1-\beta_{C}\left(\epsilon_{C}^{*}+1\right) \frac{L_{0}}{L}\right\} \\
& =\left(1-\frac{\alpha_{1}}{\alpha_{2}}\right)^{-1}\left(1-\frac{\alpha_{1}}{\alpha}\right),
\end{aligned}
$$

where $\alpha_{1} \equiv \beta_{C}\left(\epsilon_{C}^{*}+1\right)$ and $\alpha_{2} \equiv \beta_{S}\left(\epsilon_{S}+1\right)$ are materials constants. The relation between $x$ and $\alpha$ is shown in Fig. 5 together with the experimental results. One can say that Eq. (4) is qualitatively consistent with the experimental results. Here, by using the experimental values of $\beta_{C}=0.4, \beta_{S}$ $=0.8$, and $\epsilon_{C}^{*}=2, \alpha_{1}$ was determined to be 1.2 , and $\alpha_{2}$ $=2.56$ was used to obtain a good fit to the data points; by assuming $\epsilon_{S}=2.2$, which gives $E_{C} / E_{S}=4.65$. The relation,

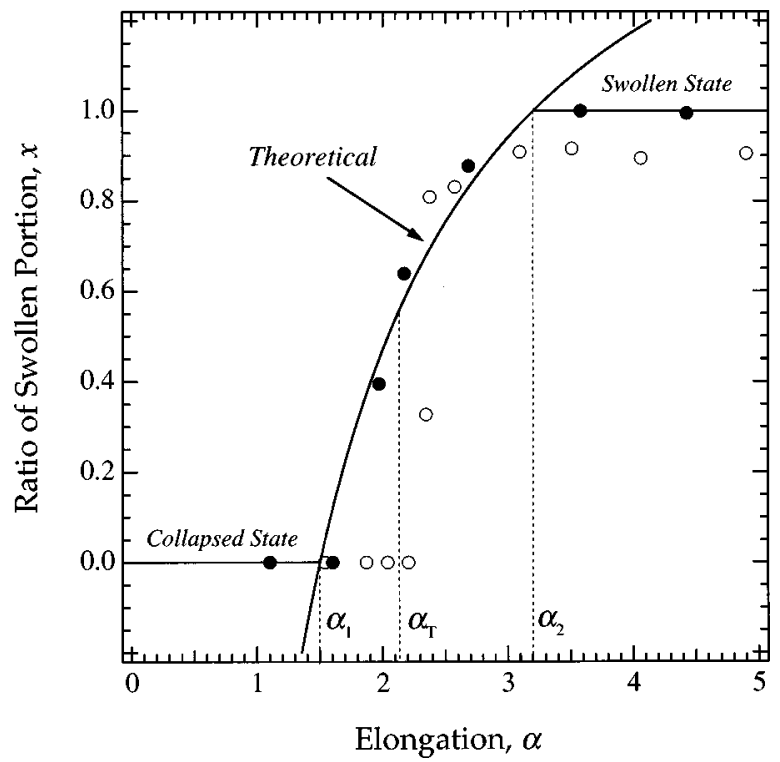

FIG. 5. Theoretical equilibrium ratio of the swollen portion to the total length, $x$ as a function of $\alpha$ at $T_{M}$ (just above the phase transition temperature), where the gel was gradually stretched along the uniaxial direction. The results are qualitatively consistent with the experimental observations.

$\alpha_{1}<\alpha_{2}$, should be satisfied to realize the phase coexistence. We estimated the condition $E_{C} / E_{S} \geqslant 3.91$ by using the above experimental values.

Figure 6 shows the $\alpha-V / V_{0}$ projection at $T_{M}$ of the three dimensional phase diagram $\left(\alpha-T-V / V_{0}\right)$. In this figure, the gel stays in the collapsed state under $\alpha \leqslant \alpha_{T}$, and in a single swollen state under $\alpha \geqslant \alpha_{2}$, while exhibiting the phase coexistence under $\alpha_{T}<\alpha<\alpha_{2}$. The ratio of the swollen to the collapsed portion can be expressed as

$$
\frac{x}{1-x}=\frac{\alpha^{-1}-\alpha_{1}^{-1}}{\alpha_{2}^{-1}-\alpha^{-1}}=\frac{\alpha_{2}}{\alpha_{1}} \frac{\alpha-\alpha_{1}}{\alpha_{2}-\alpha} \equiv \frac{\alpha_{2}}{\alpha_{1}} \frac{L_{1}}{L_{2}},
$$

where $L_{1}$ and $L_{2}$ denote the length of CQ and QR in Fig. 6, respectively. This expression corresponds to a lever rule ${ }^{27}$ for the present system: the relative length of the two phases of swollen and collapsed for a given $\alpha$ can be determined by using this phase diagram, if $\alpha_{1}$ and $\alpha_{2}$ are experimentally determined. The experimental results are qualitatively consistent with this prediction in terms of the position of the system in the coexistence curve.

\section{E. Thermally activated nucleation and phase boundary}

We have seen above that the coexistence between the swollen and the collapsed phases can be realized in the cylindrical neutral gels. Evidence for the phase coexistence can be attributed to the uniaxial constraint (isometric) during the phase transition, or to the uniaxial elongation at $T_{M}$ (a collapsed state). In both cases, the local phase transition can decrease the uniaxial stress of the remaining portion of gel, and the chains of the collapsed or swollen phase should be stretched from their equilibrium end-to-end distance at rest to the respective length with stress $\epsilon_{C}^{*}$ or $\epsilon_{S}$. In the latter case, it is interesting to observe that at smaller elongation the 


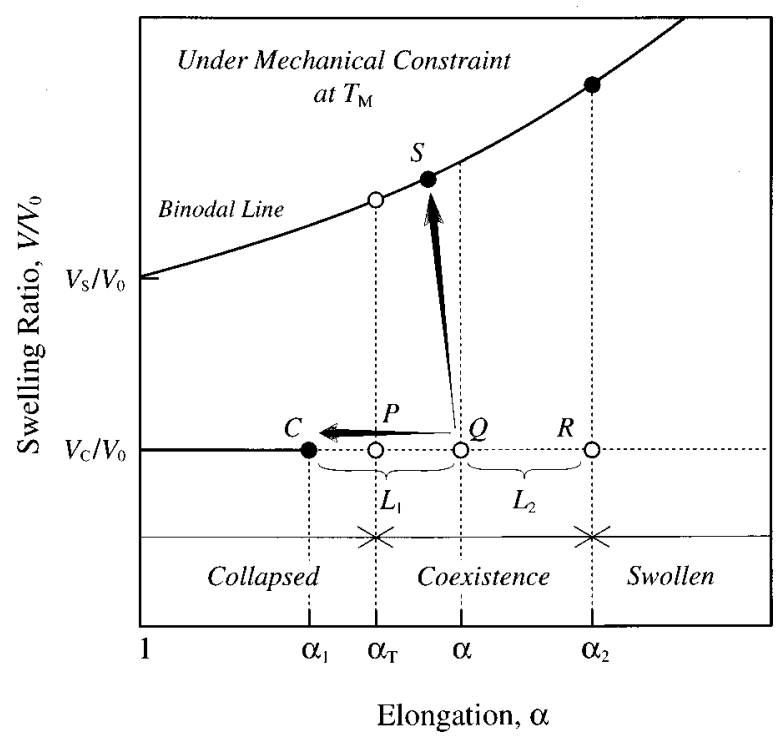

FIG. 6. Phase diagram of $\alpha-V / V_{0}$ plane at $T_{M}$, which corresponds to the phase diagram of $P$ - $\rho$ space in a fluid system. If the gel is placed at $Q$, it takes the phase coexistence of the collapsed state, $C$ and the swollen state, $S$. The ratio of the swollen to the collapsed portion is proportional to $L_{1} / L_{2}$.

whole gel stays in the collapsed state at $T_{M}$ and partly swells at larger elongation. The elongation decreases the disorientational entropy of the chains, and thus, in turn, increases the free energy which produces a restoring force. ${ }^{28,29}$ The elongation dependence of states is due to the change in the balance between hydrophobic and hydrophilic interactions of the gel and water. The water molecules in the vicinity of hydrophobic polymer chains have more ordered structures, and thus a lower entropy, than those away from the polymers. At smaller elongation the polymer network stays in the collapsed state and becomes more ordered (lower entropy), but the water molecules excluded from the polymer network become less ordered (higher entropy). At larger elongation the entropy of polymer network decreases, while that of the water molecules increases. As a whole the gel swells amount to a larger entropy of the entire gel system as should be. At the equilibrium coexistence, the restoring force is balanced in accordance with Eq. (1). In order to further understand the phase coexistence, it is important to take into consideration at least two unique elastic properties of gels under mechanical constraint. One is nucleation which is thermally activated or stress-induced, and the other is the network structures of the phase boundary.

Recent theoretical arguments ${ }^{16,30-32}$ have dealt with the fact that thermally activated nucleation is strongly suppressed in bulk gels by the distortion of the elastic field in three-dimensional gels. Nevertheless, macroscopic phase separation can experimentally occur on the gel surface, being expected to lead to the formation of a stable surface layer of the new phase. In fact, it has been experimentally pointed out that phase transition begins at the surfaces and edges of the cylindrical bulk gels, which is a consequence of long-range elastic effects. ${ }^{33,34}$ In the present simple elongation experiment, the stress can be localized in the middle of the cylindrical gel; this location is similar to that observed in the engineering fracture test of solids by tensile stress. There- fore, the initial swollen portion can emerge at the middle of the cylindrical gel. We believe that nucleation could occur everywhere in the middle portion of gels where the strain is localized. The embryos could grow to become nuclei of the new swollen phase at the critical elongation, $\alpha_{1}$. If the elongation is further increased, the nuclei would develop and combine to form a domain by decreasing the total elastic energy. When the domain can grow to the diameter size level (under $\alpha_{T} \leqslant \alpha$ ), the swollen phase can be realized in the bulk cylindrical collapsed gels. This process is expected to lead to the phase coexistence. The nucleation rate and the size of the nuclei should depend on the degree of "super-cooling", (how far the degree of elongation can exceed the critical elongation, $\alpha_{1}$ ). It should be noted that the network structure of the present kind of polymer gels is not homogeneous by nature, ${ }^{35,36}$ but rather consists of microdomains, which results in inhomogeneous strain distribution under elongation. This frozen inhomogeneities should affect the coexistence behavior. In order to verify these considerations, not only mechanical but also kinetic experiments are desirable in the same sample. This is a subject for future research.

The above theoretical consideration is based on a simple principle of mechanics which disregards the effects of the boundary between two phases. As for the phase transition of the normal gel without constraint, the diameter change is discontinuous at the transition temperature. However, at the boundary of the phase coexistence, the diameter continuously changes from one to the other. Therefore, the network in a cross-section at the boundary is expected not to become either collapsed or swollen but to coexist under the anisotropic deformation. This is because the network at the boundary is mechanically stretched in the outer surface layer but compressed in the core portion. The strain at the phase boundary should affect the total free energy of gels. ${ }^{16}$ It has been generally believed that the gel volume can be determined by osmotic pressure due to the excluded volume, which depends on polymer concentration. ${ }^{11,12}$ If the uniaxial constraint is applied, the volume is theoretically expected to change,${ }^{37}$ from our recent studies,,${ }^{17,18}$ as mentioned in the former section, the volume of a swollen phase increases with elongation, while the increment is very small in the case of a collapsed phase. This is because the repulsive interaction between polymer networks of the swollen state is much stronger than that of the collapsed state; the balance between the attractive and repulsive forces in the swollen state could be changed by the external force. In other words, the network is more hydrophilic in the swollen state that is easily affected by deformation, while in the collapsed state it is hydrophobic that is stable under deformation. From these considerations, the stress as well as the network density are highly inhomogeneous in a cross-section of the boundary, which should affect the stability of the phase coexistence. At the same time, we should consider the highly anisotropic deformation at the boundary, which results in bringing the chains closer together and thus enhancing the transverse intrachain interaction. The anisotropic stretching reduces the probability of the longitudinal interchain interaction. In particular, the transverse hydrophobic interaction on neighboring chains can be enhanced at the transition point. It is desirable to have 
microscopic information of the boundary from other techniques such as confocal microscopy, scattering experiments, and so forth under a large deformation.

We believe that these unique elastic properties of polymer gels could explain the mechanism of the discontinuous emergence of the swollen portion with a hysteresis in the present uniaxial stretch experiment. Moreover, it should be essential to understand the origin of the mechanical instability of the phase coexistence of polymer gels.

\section{CONCLUSION}

We have studied the transition behavior of cylindrical neutral poly ( $N$-isopropylacrylamide) (NIPA) gels of submillimeter diameters under a macroscopic mechanical constraint. The present investigation is focused on the phase transition behavior of neutral polymer gels in the vicinity of the transition temperature in $P-T-\rho$ space. The phase coexistence has been observed in this thermoresponsive NIPA gel by two methods. One method consists in changing the temperature under the mechanical constraint along the uniaxial direction, and the other in stretching the collapsed gel along the uniaxial direction at a constant temperature just above the transition point. The static and dynamic property of the systems to achieve the equilibrium coexistent state was investigated by optical microscope. The macroscopic mechanical constraint might change the strain distribution on the microscopic molecular level and induce a "local" phase transition within the bulk gel. Once the new phase can emerge, the remaining unchanged portion of the gel can be stabilized by being kept away from the transition point. It was also shown that the ratio of the swollen to the collapsed phase could be controlled by uniaxial stress, discussed in terms of the phase diagram (diameter vs elongation). The simple selection rule of the phase was obtained.

It can be concluded that the phase coexistence of neutral polymer gels, that is, locally collapsed or swollen state, in general, would be stable and experimentally observable when the gel exhibits the volume phase transition under a mechanical constraint. We believe that the present work is of crucial importance not only for understanding the nature of the phase transition of gels but also in establishing the foundation for the future development and application of new technologies based on these fundamental concepts.

\section{ACKNOWLEDGMENTS}

This work was supported in part by a Grant-in Aid from the Ministry of Education, Science, Sports and Culture, Japan, and by a Grant of SCF from Science and Technology Agency, Japan.

${ }^{1}$ M. E. Fisher, Rep. Prog. Phys. 30, 615 (1967).

${ }^{2}$ P. A. Egelstaff and J. W. Ring, in Physics of Simple Liquids, edited by H. N. V. Temperley, J. S. Rowlinson, and G. S. Rushbrooke (Amsterdam, North Holland, 1968), p. 253.

${ }^{3}$ H. E. Stanley, Introduction to Phase Transitions and Critical Phenomena (Oxford University Press, New York, and Oxford, 1971).

${ }^{4}$ P. J. Flory, Principle of Polymer Chemistry (Cornell University Press, Ithaca, New York, 1953).

${ }^{5}$ P.-G. de Gennes, Scaling Concepts in Polymer Physics (Cornell University Press, Ithaca and London, 1979).

${ }^{6}$ K. Dusek and D. Patterson, J. Polym. Part A-2 6, 1209 (1968).

${ }^{7}$ T. Tanaka, Phys. Rev. Lett. 40, 820 (1978).

${ }^{8}$ Y. Hirokawa and T. Tanaka, J. Chem. Phys. 81, 6379 (1984).

${ }^{9}$ A. Suzuki and T. Tanaka, Nature (London) 346, 345 (1990).

${ }^{10}$ A. Suzuki, Adv. Polym. Sci. 110, 199 (1993).

${ }^{11}$ T. Tanaka, NATO ASI Ser., Ser. E 133, 237 (1987).

${ }^{12}$ Y. Li and T. Tanaka, Annu. Rev. Mater. Sci. 22, 243 (1992).

${ }^{13}$ K. K. Lee, E. L. Cussler, M. Marchetti, and M. A. McHugh, Chem. Eng. Sci. 45, 766 (1990).

${ }^{14}$ E. Kato, Kitada, and C. Nakamoto, Macromolecules 26, 1758 (1993).

${ }^{15}$ S. Hirotsu and A. Onuki, J. Phys. Soc. Jpn. 58, 1508 (1989).

${ }^{16}$ S. Hirotsu, Phase Transit. 47, 183 (1994).

${ }^{17}$ A. Suzuki and S. Kojima, J. Chem. Phys. 101, 10003 (1994).

${ }^{18}$ A. Suzuki, K. Sanda, and Y. Omori, J. Chem. Phys. 107, 5179 (1997).

${ }^{19}$ T. Tanaka, I. Nishio, S.-T. Sun, and S. Ueno-Nishio, Science 218, 467 (1982)

${ }^{20}$ S. Hirotsu, J. Chem. Phys. 88, 427 (1988).

${ }^{21}$ S. Hirotsu, Adv. Polym. Sci. 110, 1 (1993).

${ }^{22}$ J. W. Cahn, J. Chem. Phys. 42, 93 (1965).

${ }^{23}$ R. Bansil, L. Guangdong, and P. Falus, Physica A 231, 346 (1996).

${ }^{24}$ T. Tanaka, E. Sato, Y. Hirokawa, S. Hirotsu, and J. Peetermans, Phys. Rev. Lett. 55, 2455 (1985).

${ }^{25}$ R. Becker and W. Döring, Ann. Phys. (Leipzig) 24, 719 (1935).

${ }^{26}$ K. Binder and A. P. Young, Rev. Mod. Phys. 58, 801 (1986).

${ }^{27}$ L. D. Landau and E. M. Lifshitz, Statistical Physics (Pergamon, Oxford, 1986).

${ }^{28}$ L. R. G. Treloar, The Physics of Rubber Elasticity, 2nd. ed. (Clarendon, Oxford, 1958).

${ }^{29}$ S. Candau, J. Bastide, and M. Delsanti, Adv. Polym. Sci. 44, 27 (1982).

${ }^{30}$ A. Onuki, Phys. Rev. A 38, 2192 (1988).

${ }^{31}$ K. Sekimoto, Phys. Rev. Lett. 70, 4154 (1993).

${ }^{32}$ S. Panyukov and Y. Rabin, Macromolecules 29, 8530 (1996).

${ }^{33}$ S. Hirotsu, J. Chem. Phys. 88, 427 (1988).

${ }^{34}$ E. S. Matsuo and T. Tanaka, J. Chem. Phys. 89, 1695 (1988).

${ }^{35}$ A. Suzuki, T. Ejima, Y. Kobiki, and H. Suzuki, Langmuir 13, 7039 (1997).

${ }^{36}$ E. S. Matsuo, M. Orkisz, S-T. Sun, Y. Li, and T. Tanaka, Macromolecules 27, 6791 (1994).

${ }^{37}$ P. Pekarski, A. Tkachenko, and Y. Rabin, Macromolecules 27, 7192 (1994). 
The Journal of Chemical Physics is copyrighted by the American Institute of Physics (AIP). Redistribution of journal material is subject to the AIP online journal license and/or AIP copyright. For more information, see http:/ojps.aip.org/jcpo/jcpcr/jsp Copyright of Journal of Chemical Physics is the property of American Institute of Physics and its content may not be copied or emailed to multiple sites or posted to a listserv without the copyright holder's express written permission. However, users may print, download, or email articles for individual use. 